\title{
On the Stress State of the Semi-Strip with a Longitudinal Crack
}

\author{
Natalya Vaysfeld, Zinaida Zhuravlova \\ Faculty of Mathematics, Physics and Information Technologies, Odessa I.I. Mechnikov \\ National University, Odessa, Ukraine \\ E-mail: vaysfeld@onu.edu.ua,z.zhuravlova@onu.edu.ua
}

Received: 30 September 2018; Accepted: 19 October 2018; Available online: 25 December 2018

\begin{abstract}
The stress state of the elastic semi-strip is investigated in the paper. The lateral sides of the semi-strip are fixed and the semi-strip's short edge is under the mechanical load. The longitudinal crack is located inside the semi-strip. The problem is reduced to the one-dimensional problem with the help of Fourier sin-, costransformation, which was applied directly to the Lame's equilibrium equations and the boundary conditions. The one-dimensional problem is formulated is a vector form. Its solution is constructed with the help of the matrix differential calculation and the Green matrix-function, which was constructed in the bilinear form. The solution of the problem is reduced to the solving of three singular integral equations. The first equation in this system contains two fixed singularities in its kernel. To consider them the corresponding transcendental equation is constructed, and its roots are found. The special generalized method is applied to solve the system of singular integral equations. The stress intensity factors are calculated.
\end{abstract}

Keywords: semi-strip; transverse crack; fixed singularity; singular integral equations.

\section{Introduction}

The plane elasticity problems are important as model examples for more complicated problems. The investigation of the stress state of the elastic semi-strip with a longitudinal crack can be used for the solving of the discontinuous problems in areas that contain angles and defects.

There are three classed of methods that can be applied for the solving of the plane problems of elasticity for: analytical, numeric and analytically-numeric. The analytical approaches can be used for the solving of a sufficiently narrow class of problems when the numeric approaches can be used for much more elasticity problems, however their successful application for the solving of the problems in areas that contain zones of discontinuity, such as cracks and rigid inclusions, is usually very difficult. So, the development of analytically-numeric approaches is relevant and helpful.

The plane problems of elasticity for a strip and a semi-strip were solved in the following works. The first basic odd-symmetric boundary value problem in the theory of elasticity in a half-strip with free longitudinal sides was solved in [1]. The solution was represented as series in Papkovich-Fadle eigenfunctions whose coefficients were found in an explicit form by using functions biorthogonal to the Papkovich-Fadle eigenfunctions. A method of analytical decomposition for analysis plane structures of a complex configuration was presented in [2]. For each part of the structure in the form of a rectangle all the components of the stress-strain state were constructed by the superposition method. The block element method was used to study a static boundary value problem for semiinfinite lithospheric plates interacting with a deformable basement along Conrad boundary in [3]. It was assumed that the lithospheric plates have straight line boundaries parallel to each other and are considered in two positions. In the first case, the distance between the ends of the plates did not vanish, whereas in the second case the distance was absent, although the plates do not interact.

The problems for a strips weakened by the cracks were studied in the following works. The solving of the problem for the infinite strip with a semi-infinite crack was reduced to the solving of the singular integral equation by the use of simple layer and double layer potentials in [4]. The method for the elastic strip which is weakened by cracks and holes was proposed in [5]. Non-standard boundary problem for Laplace equation in the infinite strip with a finite crack was reduced in [6] with the help of Fourier transformation to the integral equation and then to the vector Riemann-Hilbert problem.

In the proposed work the plane mixed problem for a semi-strip with a longitudinal crack was solved by the analytically-numeric approach. The integral transformations are applied directly to the Lame's equations and boundary conditions. The matrix differential apparatus and the matrix Green functions were used. The system of singular integral equations was solved with consideration of the fixed singularities in its kernel. 


\section{The statement of the problem}

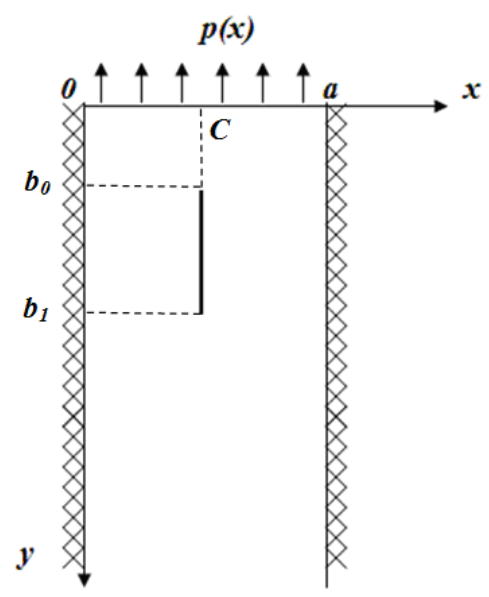

Fig. 1 Geometry and coordinate system of the semi-strip

The elastic ( $G$ is a share module, $\mu$ is a Poison ratio) semi-strip (Fig. 1), $0<x<a, 0<y<\infty$ is considered. The conditions of fixing are fulfilled on the lateral sides of the semi-strip

$$
u(0, y)=0, v(0, y)=0, u(a, y)=0, v(a, y)=0, \quad 0<y<\infty
$$

where $u(x, y)=u_{x}(x, y), v(x, y)=u_{y}(x, y)$ are the displacement functions that satisfy the Lame's equilibrium equations:

$$
\left\{\begin{array}{l}
\frac{\partial^{2} u(x, y)}{\partial x^{2}}+\frac{\kappa-1}{\kappa+1} \frac{\partial^{2} u(x, y)}{\partial y^{2}}+\frac{2}{\kappa+1} \frac{\partial^{2} v(x, y)}{\partial x \partial y}=0 \\
\frac{\partial^{2} v(x, y)}{\partial x^{2}}+\frac{\kappa+1}{\kappa-1} \frac{\partial^{2} v(x, y)}{\partial y^{2}}-\frac{2}{\kappa-1} \frac{\partial^{2} u(x, y)}{\partial x \partial y}=0
\end{array}\right.
$$

here $\kappa=3-4 \mu$ is the Muskchelishvili constant.

The semi-strip is loaded at its short edge

$$
\sigma_{y}(x, 0)=p(x), \quad \tau_{x y}(x, 0)=0,0<x<a
$$

The longitudinal crack is located inside the semi-strip on the line $b_{0}<y<b_{1}, x=C$

$$
\begin{gathered}
u(C-0, y)-u(C+0, y)=\langle u(C, y)\rangle=\varphi_{1}(y) \neq 0, b_{0}<y<b_{1} \\
v(C-0, y)-v(C+0, y)=\langle v(C, y)\rangle=\varphi_{2}(y) \neq 0, b_{0}<y<b_{1} \\
\tau_{x y}(C-0, y)-\tau_{x y}(C+0, y)=\left\langle\tau_{x y}(C, y)\right\rangle=0, b_{0}<y<b_{1} \\
\sigma_{x}(C-0, y)-\sigma_{x}(C+0, y)=\left\langle\sigma_{x}(C, y)\right\rangle=0, b_{0}<y<b_{1} \\
\left.\tau_{x y}\right|_{x=C \pm 0}=0,\left.\quad \sigma_{x}\right|_{x=C \pm 0}=p_{\sigma}(y), \quad b_{0}<y<b_{1}
\end{gathered}
$$

The problem (1)-(5) should be solved to estimate the stress state of the semi-strip.

\section{The general solving scheme}

The initial problem (1)-(5) is reduced to the one-dimensional problem with the help of Fourier sin-, costransformation applied by the variable $y$ [7].

The following vectors and matrices are inputted

$$
\begin{gathered}
\vec{y}_{\beta}(x)=\left(\begin{array}{c}
u_{\beta}(x) \\
v_{\beta}(x)
\end{array}\right), \\
\vec{f}(x)=\left(\begin{array}{c}
\frac{3-\kappa}{\kappa+1} \chi^{\prime}(x) \\
-\beta \frac{\kappa+1}{\kappa-1} \chi(x)
\end{array}\right), \\
N_{\beta}^{0}=\left(\begin{array}{c}
\varphi_{1 \beta} \\
\varphi_{2 \beta}
\end{array}\right), N_{\beta}^{1}=\left(\begin{array}{c}
\beta \frac{\kappa-3}{\kappa+1} \varphi_{2 \beta} \\
\beta \varphi_{1 \beta}
\end{array}\right), \\
P=\left(\begin{array}{cc}
\frac{\kappa-1}{\kappa+1} & 0 \\
0 & \frac{\kappa+1}{\kappa-1}
\end{array}\right),
\end{gathered}
$$




$$
Q=\left(\begin{array}{cc}
0 & \frac{1}{\kappa+1} \\
-\frac{1}{\kappa-1} & 0
\end{array}\right)
$$

Here $\chi(x)=\left.v(x, y)\right|_{y=0}$ is the unknown function.

The vector problem in the transformation domain is formulated as following

$$
\left\{\begin{array}{c}
L_{2} \vec{y}_{\beta}(x)=\vec{f}(x), \\
\vec{y}_{\beta}(0)=0, \vec{y}_{\beta}(a)=0, \\
\left\langle\vec{y}_{\beta}(C)\right\rangle=N_{\beta}^{0},\left\langle\vec{y}_{\beta}^{\prime}(C)\right\rangle=N_{\beta}^{1}
\end{array}\right.
$$

The solution of the problem (6) is constructed as the superposition of the general solution of the corresponding homogeneous equation, the partial solution of the inhomogeneous equation and the discontinuous solution of the problem [8].

\subsection{The construction of the general solution}

The general solution of the homogeneous vector equation is found with the help of the corresponding matrix equation $L_{2} Y(x)=0,0<x<a$, which solution is found by the formula $Y(x)=\frac{1}{2 \pi i} \oint_{C} e^{\xi x} M^{-1}(\xi) d \xi$, where $M(\xi)=I \xi^{2}+2 \beta Q \xi-\beta^{2} P$. So the general solution of the homogeneous vector equation has the following form $\vec{y}_{\beta}^{0}(x)=Y_{1}(x)\left(\begin{array}{l}c_{1} \\ c_{2}\end{array}\right)+Y_{2}(x)\left(\begin{array}{l}c_{3} \\ c_{4}\end{array}\right)$, where $Y_{1}(x), Y_{2}(x)$ are the fundamental matrix solutions, $c_{i}, i=\overline{1,4}$ are known constants.

\subsection{The construction of the partial and discontinuous solutions}

The following problem is considered

where

$$
\left\{\begin{array}{c}
L_{2} \vec{y}_{\beta}(x)=\vec{f}(x), \\
V_{i}[\vec{y}(x)]=0, i=0,1, \\
\left\langle\vec{y}_{\beta}(C)\right\rangle=N_{\beta}^{0},\left\langle\vec{y}_{\beta}^{\prime}(C)\right\rangle=N_{\beta}^{1}
\end{array}\right.
$$

The matrix integral transformation with the kernel

$$
V_{0}[\vec{y}(x)]=\alpha_{n}\left(\begin{array}{ll}
1 & 0 \\
0 & 0
\end{array}\right) \vec{y}(0)-\left(\begin{array}{ll}
0 & 0 \\
0 & 1
\end{array}\right) \vec{y}^{\prime}(0), V_{1}[\vec{y}(x)]=\alpha_{n}\left(\begin{array}{ll}
1 & 0 \\
0 & 0
\end{array}\right) \vec{y}(a)-\left(\begin{array}{ll}
0 & 0 \\
0 & 1
\end{array}\right) \vec{y}^{\prime}(a) .
$$

$$
H\left(x, \alpha_{n}\right)=\left(\begin{array}{cc}
\sin \alpha_{n} x & 0 \\
0 & \cos \alpha_{n} x
\end{array}\right), \alpha_{n}=\frac{n \pi}{a}, n=0,1,2 \ldots
$$

is applied to the problem (7) by the generalized scheme [9].

The problem (7) in the transformation domain can be written as $\Omega_{\beta}\left(\alpha_{n}\right) \vec{y}_{n}=\vec{F}_{n}$.

Here

$$
\begin{gathered}
\Omega_{\beta}\left(\alpha_{n}\right)=-I \alpha_{n}^{2}-2 \beta \alpha_{n} \tilde{Q}-\beta^{2} P, \\
\tilde{Q}=\left(\begin{array}{cc}
0 & \frac{1}{\kappa+1} \\
\frac{1}{\kappa-1} & 0
\end{array}\right), \\
\vec{y}_{n}=\int_{0}^{a} \vec{y}(x) H\left(x, \alpha_{n}\right) d x, \vec{F}_{n}=\vec{f}_{n}+\vec{\Phi}_{n}, \\
\Phi_{n}=\left(\begin{array}{c}
\alpha_{n} \cos \left(\alpha_{n} c\right) \varphi_{1 \beta}-\beta \frac{\kappa-1}{\kappa+1} \sin \left(\alpha_{n} c\right) \varphi_{2 \beta} \\
-\beta \frac{\kappa-3}{\kappa-1} \cos \left(\alpha_{n} c\right) \varphi_{1 \beta}-\alpha_{n} \sin \left(\alpha_{n} c\right) \varphi_{2 \beta}
\end{array}\right) .
\end{gathered}
$$

After the inverting of the integral transformation the partial $\vec{y}_{\beta}^{1}(x)=\int_{0}^{a} G(x, \xi) \vec{f}(\xi) d \xi$ and the discontinuous $\vec{y}_{\beta}^{p}(x)=\frac{2}{a} \sum_{n=0}^{\infty} H\left(x, \alpha_{n}\right) \Omega_{\beta}^{-1}\left(\alpha_{n}\right) \vec{\Phi}_{n}$ solutions are found. Here $G(x, \xi)=\frac{2}{a} \sum_{n=0}^{\infty}{ }^{\prime} H\left(x, \alpha_{n}\right) \Omega_{\beta}^{-1}\left(\alpha_{n}\right) H\left(\xi, \alpha_{n}\right)$ is Green matrix-function which is constructed in the bilinear form.

\section{The solving of the singular integral equations}

The expressions for the displacement functions contain three unknown functions $\chi(x), \varphi_{1}(y), \varphi_{2}(y)$. For its finding the following system of singular integral equations (SSIE) is derived 


$$
\left\{\begin{array}{c}
\int_{-1}^{1} \hat{\chi}(\xi)\left[\frac{1}{\xi-x}+Z_{2}(x, \xi)\right] d \xi+\widehat{K}_{0}(x)=\hat{r}(x), x \in I_{1} \\
\frac{d^{2}}{d y^{2}} \int_{-1}^{1} \tilde{\varphi}_{1}(\eta) \ln \frac{1}{|\eta-y|} d \eta+\widehat{K}_{1}(y)=0, y \in I_{1} \\
\frac{d^{2}}{d y^{2}} \int_{-1}^{1} \tilde{\varphi}_{2}(\eta) \ln \frac{1}{|\eta-y|} d \eta+\widehat{K}_{2}(y)=0, y \in I_{1}
\end{array}\right.
$$

with the additional conditions $\int_{-1}^{1} \hat{\chi}(\xi) d \xi=0$.

Here

$$
\begin{gathered}
\hat{\chi}(\xi)=\chi^{\prime}\left(\frac{a(\xi+1)}{2}\right), \\
Z_{2}(x, \xi)=h_{1}\left(\frac{1}{\xi+x-2}+\frac{1}{\xi+x+2}\right)+h_{2}\left(\frac{x-1}{(\xi+x-2)^{2}}+\frac{x+1}{(\xi+x+2)^{2}}\right)+h_{3}\left(\frac{(\xi-1)(x-1)}{(\xi+x-2)^{3}}+\frac{(\xi+1)(x+1)}{(\xi+x+2)^{3}}\right), \\
h_{1}=-\frac{\kappa^{2}-3}{2 \kappa}, h_{2}=-\frac{2}{\kappa}, h_{3}=\frac{4}{\kappa}, \\
\tilde{\varphi}_{i}(\eta)=\varphi_{i}\left(\frac{\left(b_{1}-b_{0}\right) \eta+\left(b_{1}+b_{0}\right)}{2}\right), i=1,2, \\
\widehat{K}_{0}(x)=\int_{-1}^{1} \hat{\chi}(\xi) \hat{f}_{0}(\xi, x) d \xi+\int_{-1}^{1} \tilde{\varphi}_{1}(\eta) \hat{R}_{0,1}(+0, \eta) d \eta+\int_{-1}^{1} \tilde{\varphi}_{2}(\eta) \hat{R}_{0,2}(+0, \eta) d \eta, \\
\widehat{K}_{i}(y)=\int_{-1}^{1} \hat{\chi}(\xi) \hat{f}_{i}(\xi, C+0) d \xi+\int_{-1}^{1} \tilde{\varphi}_{1}(\eta) \hat{R}_{i, 1}(y, \eta) d \eta+\int_{-1}^{1} \tilde{\varphi}_{2}(\eta) \hat{R}_{i, 2}(y, \eta) d \eta, i=1,2 \\
\hat{f}_{i}(\xi, x), \hat{R}_{i, 1}(y, \eta), \hat{R}_{i, 2}(y, \eta), \hat{r}(x), i=0,1,2
\end{gathered}
$$

are known regular functions.

The first equation in (8) contains two fixed singularities. The corresponding transcendental equation is constructed. It is congruent to the transcendental equation presented in [10] for the problem of the wedge with the angle of openness $\pi / 2$. The roots $\lambda_{k}$ of the transcendental equation are found numerically.

The generalized method [11] is used for the solving of SSIE (8). Accordingly to it the unknown functions are searched in the form

where

$$
\hat{\chi}(\xi)=\sum_{k=0}^{N-1}\left[s_{k} \rho_{k}^{-}(\xi)+s_{k+N} \rho_{k}^{+}(\xi)\right], \xi \in[-1 ; 1]
$$

$$
\begin{gathered}
\rho_{2 k}^{\mp}(\xi)=(1 \pm \xi)^{R e \lambda_{k}} \cdot \cos \left(\operatorname{Im} \lambda_{k} \ln (1 \pm \xi)\right), \quad k=\overline{0, N-1} . \\
\rho_{2 k+1}^{\mp}(\xi)=(1 \pm \xi)^{R e} \lambda_{k} \cdot \sin \left(\operatorname{Im} \lambda_{k} \ln (1 \pm \xi)\right), \\
\tilde{\varphi}_{i}(\eta)=\sum_{n=0}^{2 N-1} s_{n}^{i} \sqrt{1-\eta^{2}} U_{n}(\eta), \eta \in[-1 ; 1] i=1,2
\end{gathered}
$$

here $U_{n}(y)$ are Chebyshev polynomials of the second kind.

The segment $[-1 ; 1]$ is divided on $2 N$ segments by the points $x_{i}: P_{2 N-1}^{\lambda_{0},-0.5}\left(x_{i}\right)=0, i=\overline{0,2 N-1}$. SSIE (20) is considered when $x=x_{i}, i=\overline{0,2 N-1}$. The system linear algebraic equations is obtained

$$
\sum_{n=0}^{\infty} D_{m n} \vec{S}_{n}=\vec{f}_{m}, m=\overline{0,2 N-1}
$$

where $\vec{S}_{m}=\left(s_{m}^{0} ; s_{m}^{1} ; s_{m}^{2}\right)^{T}$, and components of $D_{m n}=\left\{d_{m n}^{i j}\right\}, i, j=0,1,2, \vec{f}_{m}=\left(f_{m}^{0} ; f_{m}^{1} ; f_{m}^{2}\right)^{T}$ are known constants.

\section{The stress intensity factors}

The stress intensity factors can be found as [12]

$$
\begin{gathered}
K_{I-}=\sum_{k=0}^{2 N-1} s_{k}^{1} \frac{\sqrt{\pi\left(b_{1}-b_{0}\right)}(n+1)(-1)^{k}}{\sqrt{2}}, K_{I+}=\sum_{k=0}^{2 N-1} s_{k}^{1} \frac{\sqrt{\pi\left(b_{1}-b_{0}\right)}(n+1)}{\sqrt{2}} \\
K_{I I-}=\sum_{k=0}^{2 N-1} s_{k}^{2} \frac{\sqrt{\pi\left(b_{1}-b_{0}\right)}(n+1)(-1)^{k}}{\sqrt{2}}, K_{I+}=\sum_{k=0}^{2 N-1} s_{k}^{2} \frac{\sqrt{\pi\left(b_{1}-b_{0}\right)}(n+1)}{\sqrt{2}}
\end{gathered}
$$

\section{Conclusions}

The new approach was used for the solving of the plane mixed elasticity problem for a semi-strip. The partial and discontinuous solutions were constructed with the help of the integral transformation applied by the generalized scheme. The solving of the problem was reduced to the solving of SSIE, where the first equation contains two fixed singularities. The corresponding transcendent equation was constructed and its roots were found. SSIE was solved with the help of the generalized method. 


\section{References}

[1] Kovalenko, M.D., Menshova, I.V., Kerzhaev, A.P. On the exact solutions of the biharmonic problem of the theory of elasticity in a half-strip. Zeitschrift fur Angewandte Mathematik und Physik. Vol. 69 (2018), Issue 5.

[2] Goloskokov, D.P., Matrosov, A.V. Approximate solutions in the analysis of elastic structures of complex geometry. AIP Conference Proceedings. Vol. 1959 (2018).

[3] Babeshko, V.A., Evdokimova, O.V., Babeshko, O.M. On a mechanical approach to the prediction of earthquakes during horizontal motion of lithospheric plates. Acta Mechanica. 2018.

[4] Itou, H., Tani, A. A boundary value problem for an infinite elastic strip with a semi-infinite crack. Journal of Elasticity. Vol. 66 (2002), p. 193-206.

[5] Theotokoglou, E. N., Tsamasphyros, G. J. Integral equations for any configuration of cured cracks and holes in an elastic strip. Ingenieur-Archiv. Vol. 57 (1987), p. 3-15.

[6] Antipov, Y.A., Schiavone, P. Integro-differential equation of a finite crack in a strip with surface effects. Quart. J. Mech. Appl. Math. Vol. 64 (2011), p. 87-106.

[7] Vaysfel'd, N.D., Zhuravlova, Z.Yu. Two-dimensional mixed problem of thermoelasticity for a semistrip. Journal of Mathematical Sciences. Vol. 228 (2018), Issue 2, p. 105-121. DOI 10.1007/s10958-017-3609-8.

[8] Vaysfel'd, N.D., Zhuravlova, Z.Yu. On one new approach to the solving of an elasticity mixed problem for the semi-strip. Acta Mechanica. Vol. 226 (2015), Issue 12, p. 4159-4172. DOI: 10.1007/s00707-015-1452-X.

[9] Popov, G.Ya. The elastic stress' concentration around dies, cuts, thin inclusions and reinforcements. Nauka, Moskow. 1982. (in Russian)

[10] Uflyand, Ya.S. Integral transformations in the problems of the elasticity theory. Nauka, L. 1967. (in Russian) [11] Reut, V., Vaysfeld, N., Zhuravlova, Z. Elastic crack-tip stress field in a semi-strip. Frattura ed Integrità Strutturale. Vol. 44 (2018), p. 82-93. DOI: 10.3221/IGF-ESIS.44.07

[12] Zhuravlova, Z. Stress analysis near the tips of a transverse crack in an elastic semi-strip. Appl. Math. Mech. -Engl. Ed. 2017. DOI 10.1007/s10483-017-2217-6

(C) 2018 by the author(s). This work is licensed under a Creative Commons Attribution 4.0 International License (http://creativecommons.org/licenses/by/4.0/). Authors retain copyright of their work, with first publication rights granted to Tech Reviews Ltd. 